\title{
Ascending aorta and aortic arch pseudoanaeurysm case report
}

\author{
Miroslav Krpan, Ljiljana Banfić, Majda Vrkić Kirhmajer, Krešimir Putarek, Zoran Miovski \\ University of Zagreb School of Medicine, University Hospital Centre Zagreb, Zagreb, Croatia
}

We present a 53-year-old patient who was admitted with high fever, cough and nasal discharge. She received her kidney transplant ten years ago with subsequent taking of common immunosupressive therapy with several past hospitalizations due to respiratory infections and respiratory failure. Twenty years ago, she underwent an urgent neurosurgical procedure due to subarachnoid bleeding with underlying ruptured intracranial anaeurysm. One month before actual hospitalization, the patient was complaining of chest pain. Diagnostic work-up of the infection included native computerised tomography (CT) of the thorax and abdomen with coincidental finding of the ascending aorta and aortic arch anaeurysm. Subsequent CT aortography with reconstructions was performed showing pseudoanaeurysm of the ascending aorta and aortic arch $(6.2 \times 2.7 \mathrm{~cm})$ anteriorly and

\section{Received: $20^{\text {th }}$ Apr 2014}

*Address for correspondence: Klinički bolnički centar Zagreb, Kišpatićeva 12, HR10000 Zagreb, Croatia.

Phone: 385-98-1710-329

Email: ljiljanabanfic@yahoo.com laterally of the ascending aorta and arch until the origin of the left common carotid and left subclavian artery with wide communication of the aorta and pseudoanaeurysm. Anaeurysmatic dilatation of the splenic artery was diagnosed as well $(2.6 \mathrm{~cm})$.

Cardiothoracic operation was planned and coronary angiography (transfemoral) was performed as well with the normal finding of the epicardial coronary arteries but with postprocedural haemorrhagic complication with large ipsilateral haematoma of the rectus abdominis and retroperitoneum requiring percutaneous occlusion with BeadBlock Terumo spheric particles of the inferior epigastric artery with an optimal result. Several heamodialysis procedures were undertaken after all contrast imaging procedures in order to protect the transplanted renal graft. Due to a blood loss, the patient was transfused with seven units of blood altogether. She was treated with meropenem and is afebrile with good general condition pending operation of the aorta.

KEWWORDS: pseudoanaeurysm, aorta, ascending, arch. CITATION: Cardiol Croat. 2014;9(5-6):203.

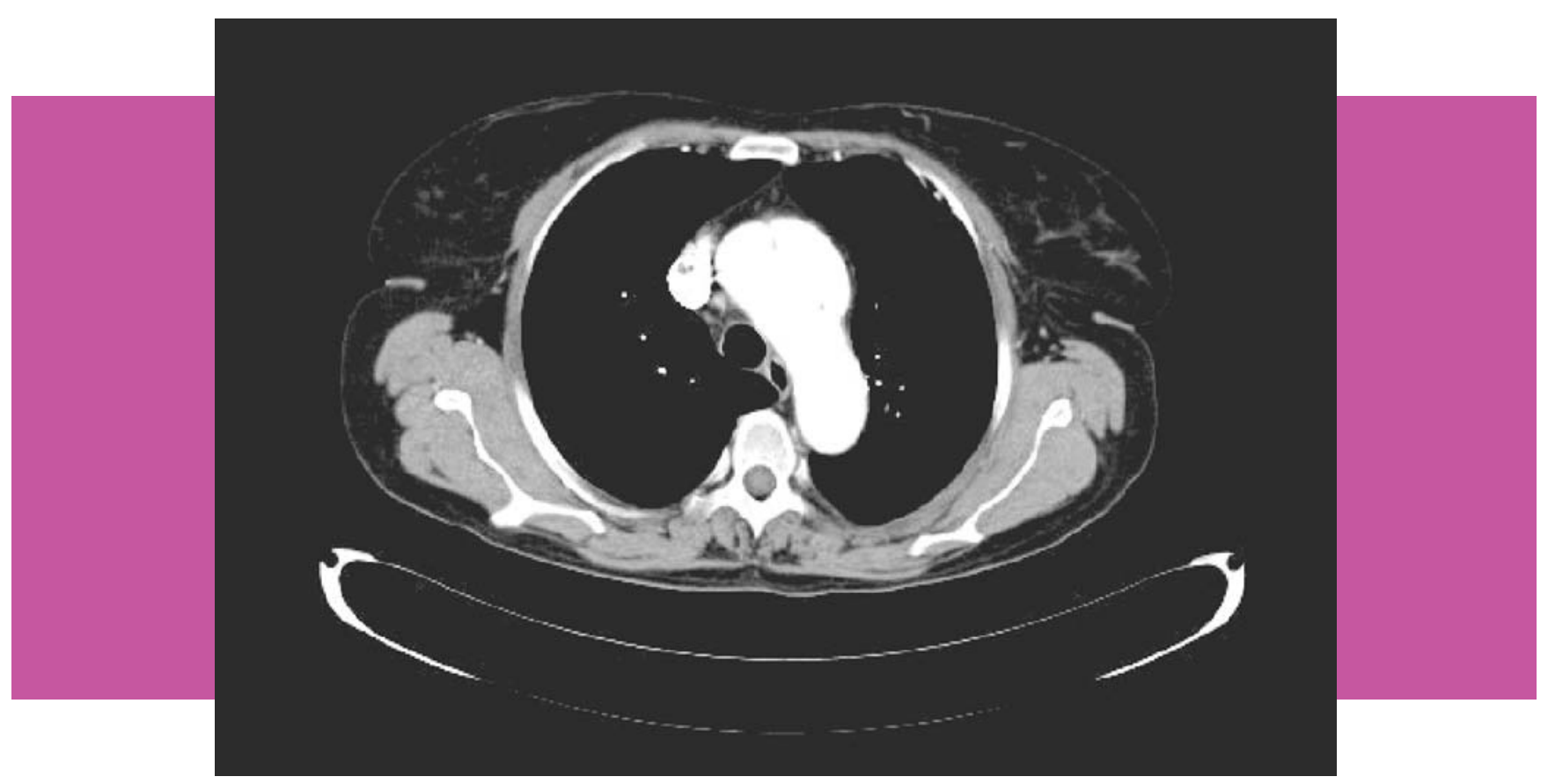

Figure 1. Computerized tomography aortography showing pseudoanaeurysm anteriorly to the ascending aorta and aortic arch.. 